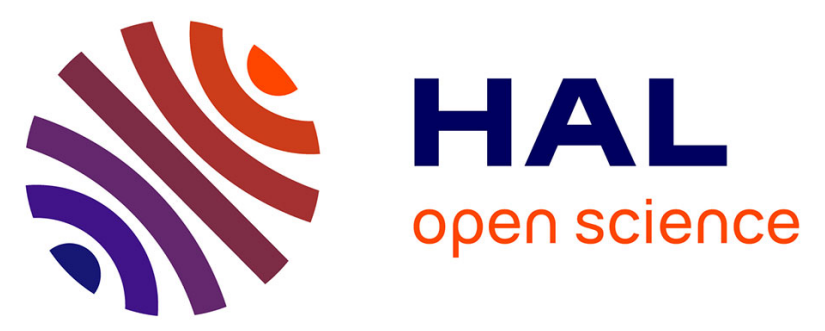

\title{
Low noise all-oxide magnetic tunnel junctions based on a La_0.7Sr_0.3Mn_O3/Nb:SrTiO_3 interface
}

Georges Kurij, Aurélie Solignac, T. Maroutian, G. Agnus, R. Guerrero, L.E. Calvet, Myriam Pannetier-Lecoeur, Philippe Lecoeur

\section{- To cite this version:}

Georges Kurij, Aurélie Solignac, T. Maroutian, G. Agnus, R. Guerrero, et al.. Low noise all-oxide magnetic tunnel junctions based on a La_0.7Sr_0.3Mn_O3/Nb:SrTiO_3 interface. Applied Physics Letters, 2017, 110, pp.82405. 10.1063/1.4977173 . cea-01503092

\section{HAL Id: cea-01503092 https://hal-cea.archives-ouvertes.fr/cea-01503092}

Submitted on 6 Apr 2017

HAL is a multi-disciplinary open access archive for the deposit and dissemination of scientific research documents, whether they are published or not. The documents may come from teaching and research institutions in France or abroad, or from public or private research centers.
L'archive ouverte pluridisciplinaire HAL, est destinée au dépôt et à la diffusion de documents scientifiques de niveau recherche, publiés ou non, émanant des établissements d'enseignement et de recherche français ou étrangers, des laboratoires publics ou privés. 


\section{Low noise all-oxide magnetic tunnel junctions based on a $\mathrm{La}_{0.7} \mathrm{Sr}_{0.3} \mathrm{MnO}_{3} / \mathrm{Nb}_{\mathrm{SrTiO}}$ interface}

G. Kurij, A. Solignac, T. Maroutian, G. Agnus, R. Guerrero, L. E. Calvet, M. Pannetier-Lecoeur, and Ph. Lecoeur

Citation: Appl. Phys. Lett. 110, 082405 (2017); doi: 10.1063/1.4977173

View online: http://dx.doi.org/10.1063/1.4977173

View Table of Contents: http://aip.scitation.org/toc/apl/110/8

Published by the American Institute of Physics

\section{Articles you may be interested in}

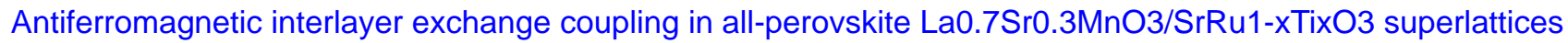
Appl. Phys. Lett. 110, 082402082402 (2017); 10.1063/1.4976509

Room temperature insulating ferromagnetism induced by charge transfer in ultrathin (110) La0.7Sr0.3MnO3 films

Appl. Phys. Lett. 110, 072405072405 (2017); 10.1063/1.4976699

Interface induced out-of-plane magnetic anisotropy in magnetoelectric BiFeO3-BaTiO3 superlattices

Appl. Phys. Lett. 110, 092902092902 (2017); 10.1063/1.4977434

Electric field effect near the metal-insulator transition of a two-dimensional electron system in SrTiO3 Appl. Phys. Lett. 110, 062104062104 (2017); 10.1063/1.4975806

Multiferroic heterostructures of Fe3O4/PMN-PT prepared by atomic layer deposition for enhanced interfacial magnetoelectric couplings

Appl. Phys. Lett. 110, 082902082902 (2017); 10.1063/1.4976008

Ultra-low magnetic damping of perovskite La0.7Sr0.3MnO3 thin films

Appl. Phys. Lett. 110, 112401112401 (2017); 10.1063/1.4978431
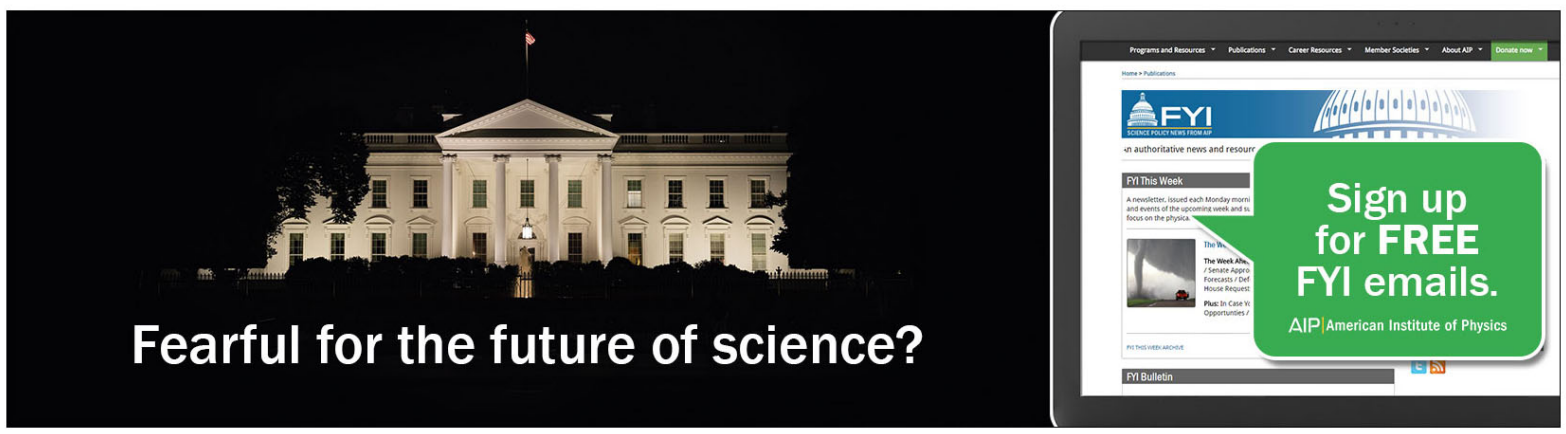


\title{
Low noise all-oxide magnetic tunnel junctions based on a $\mathrm{La}_{0.7} \mathrm{Sr}_{0.3} \mathrm{MnO}_{3} /$ $\mathrm{Nb}: \mathrm{SrTiO}_{3}$ interface
}

\author{
G. Kurij, ${ }^{1, a)}$ A. Solignac, ${ }^{2}$ T. Maroutian, ${ }^{1}$ G. Agnus, ${ }^{1}$ R. Guerrero, ${ }^{1}$ L. E. Calvet, ${ }^{1}$ \\ M. Pannetier-Lecoeur, ${ }^{2}$ and Ph. Lecoeur ${ }^{1}$ \\ ${ }^{1}$ Centre de Nanosciences et de Nanotechnologies, CNRS UMR 9001, Univ. Paris-Sud, Université Paris-Saclay, \\ C2N - Orsay, 91405 Orsay, France \\ ${ }^{2}$ SPEC, CEA, CNRS, Université Paris-Saclay, CEA Saclay, 91191 Gif-sur-Yvette, France
}

(Received 19 December 2016; accepted 10 February 2017; published online 24 February 2017)

\begin{abstract}
All-oxide magnetic tunnel junctions with a semiconducting barrier, formed by the half-metallic ferromagnet $\mathrm{La}_{0.7} \mathrm{Sr}_{0.3} \mathrm{MnO}_{3}$ and n-type semiconductor $\mathrm{SrTi}_{0.8} \mathrm{Nb}_{0.2} \mathrm{O}_{3}$, were designed, fabricated, and investigated in terms of their magneto-transport properties as a function of applied bias and temperature. We found that the use of the heavily $\mathrm{Nb}$-doped $\mathrm{SrTiO}_{3}$ as a barrier results in significant improvement in the reproducibility of results, i.e., of large tunnel magnetoresistance (TMR) ratios, and a spectral noise density reduced by three orders of magnitude at low temperature. We attribute this finding to a considerably decreased amount of point defects in $\mathrm{SrTi}_{0.8} \mathrm{Nb}_{0.2} \mathrm{O}_{3}$, especially oxygen vacancies, compared with the conventional insulating $\mathrm{SrTiO}_{3}$ barrier. Published by AIP Publishing. [http://dx.doi.org/10.1063/1.4977173]
\end{abstract}

Functional oxides represent a key class of materials for future applications due to a wide range of their physical properties.

In particular, fully spin polarized manganite $\mathrm{La}_{0.67} \mathrm{Sr}_{0.33}$ $\mathrm{MnO}_{3}$ (LSMO) ${ }^{1,2}$ has attracted significant interest for applications in magnetic field sensors. However, despite large tunnel magnetoresistance ratios (TMRs), LSMO based magnetic tunnel junctions (MTJs) often exhibit a lack of reproducibility and a noise level in the low frequency range that is always higher than in metallic MTJs. ${ }^{3}$

The limits in performance of $\mathrm{LSMO}_{\mathrm{SrTiO}}$ (STO)STO/ LSMO tunnel junctions are often attributed to defects in the barrier, such as oxygen vacancies created during the thin film deposition, or reduced spin polarization in LSMO at the interface with STO. ${ }^{4}$ This so-called "dead layer" was shown to be responsible for the loss of magnetoresistance with increasing temperature, far from the Curie temperature of LSMO.

In light of these considerations, a reduction of the oxygen vacancy content in the tunnel barrier and at the electrodebarrier interface should lead to improved overall performance of the tunnel junction. To achieve this, we replaced the standard insulating STO tunnel barrier in LSMO MTJs by a niobium-doped STO (Nb:STO), which is an n-type semiconductor. $^{5}$ In contact with LSMO, Nb:STO forms a metalsemiconductor interface, providing a structure similar to a Schottky junction. ${ }^{6,7}$ Thus, a different type of STO-based tunnel junction arises: MTJ with a double Schottky barrier instead of the conventional insulating tunnel barrier. The use of a semiconducting barrier can be traced back to the pioneering work of M. Jullière. ${ }^{8}$ Opting for a wide band gap semiconducting oxide has recently shown large TMR values at room temperature. ${ }^{9}$ Here, we show that a similar scheme, applied to an all-oxide junction, will have two main impacts. First, the substitution of $\mathrm{Nb}^{5+}$ for $\mathrm{Ti}^{4+}$ in STO results in the compound

\footnotetext{
${ }^{\text {a) }}$ Author to whom correspondence should be addressed. Electronic mail: georg.kurij@u-psud.fr
}

$\mathrm{SrTi}_{1-\mathrm{x}} \mathrm{Nb}_{\mathrm{x}} \mathrm{O}_{3}$. The niobium ions (donor centres) and the oxygen vacancies are positively charged. We hypothesize that niobium doping drastically reduces the number of oxygen vacancies during thin film growth due to charge conservation. A possible reduction of the oxygen content by means of niobium doping has been reported. ${ }^{10,11}$ Second, the origin of tunnel transport through the double Schottky barrier is different from the standard insulating tunnel barrier.

In this letter, we report temperature- and voltagedependent magneto-transport measurements on LSMO based tunnel junctions with a semiconducting $\mathrm{Nb}$ :STO barrier and compare the results with those obtained on MTJs of the same composition but with an undoped STO barrier, fabricated with the same methods and on the same equipment, reported in Ref. 3. We find evidence that $\mathrm{Nb}$ doping indeed provides an enhanced quality of the tunnel barrier, resulting in the improved reproducibility of large TMR ratios, with TMR present up to room temperature and strongly reduced noise levels.

Multilayers with the composition $\operatorname{LSMO}(30 \mathrm{~nm}) /$ $\mathrm{Nb}: \mathrm{STO} / \mathrm{LSMO}(10 \mathrm{~nm}) / \mathrm{La}_{0.7} \mathrm{Sr}_{0.3} \mathrm{Mn}_{0.93} \mathrm{Ru}_{0.07} \mathrm{O}_{3}$ (LSMRO, $20 \mathrm{~nm}$ ) were grown in situ by pulsed laser deposition on $\mathrm{TiO}_{2}$ single-terminated, (100)-oriented $\mathrm{SrTiO}_{3}$ substrates. The Nb:STO layer thickness in our junctions was varied between $1.8 \mathrm{~nm}$ and $3.0 \mathrm{~nm}$. In this paper, we present the results obtained on the sample with a $2.6 \mathrm{~nm}$ barrier, which exhibited the largest TMR ratio of all. LSMRO has an increased coercivity compared to LSMO due to ruthenium $(\mathrm{Ru})$ doping and was used as the hard layer. ${ }^{12}$ The additional $10 \mathrm{~nm}$-thick LSMO layer helps to avoid Ru diffusion into the barrier. We used a $\mathrm{KrF}$ excimer laser with $248 \mathrm{~nm}$ wavelength and $2 \mathrm{~Hz}$ repetition rate. The substrate temperature was held at $600{ }^{\circ} \mathrm{C}$ and the oxygen background pressure at 120 mTorr for LSMO and LSMRO and 12 mTorr for $\mathrm{Nb}$ :STO. After deposition, the multilayers were cooled down at 75 Torr of pure oxygen atmosphere. We analyzed surface topography and crystallographic properties of our samples by means of atomic force microscopy and X-ray diffraction 
(Fig. 1(b)). Layer thicknesses were obtained using X-ray reflectivity.

Tunnel junctions (Fig. 1(a)) with mesa dimensions ranging from $5 \times 5 \mu \mathrm{m}^{2}$ to $10 \times 10 \mu \mathrm{m}^{2}$ were fabricated using photolithography and chemically assisted ion beam etching controlled by a secondary ion mass spectrometer. We used sputtering to deposit palladium contact pads. Junctions were connected by wire bonding. We performed magnetotransport measurements in the $20-300 \mathrm{~K}$ temperature range, with a magnetic field applied in the plane of the films. Measurements in both two-point- and four-point-configurations provided the same result due to negligible contact resistance. Either voltage or current sources were used without noticeably modifying the TMR ratio. In all cases, the signal is amplified by a low noise amplifier and recorded with an acquisition card. The resistance area products $(R A)$ are typically around $100 \mathrm{M} \Omega \cdot \mu \mathrm{m}^{2}$.

Fig. 1(c) shows the magnetoresistance variation of the MTJ with a TMR ratio of $350 \%$ recorded at $20 \mathrm{~K}$ down to $100 \%$ at $100 \mathrm{~K}$. Here, the TMR ratio is defined as TMR $=\left(R_{A P}-R_{P}\right) / R_{P}$, where $R_{A P}$ and $R_{P}$ are the antiparallel and parallel resistances, respectively. These values and the general behaviour are compared with those obtained for similar MTJs with an undoped STO barrier of $3 \mathrm{~nm}$ thickness, grown, and fabricated on the same equipment. ${ }^{13}$ A striking feature of Nb:STO-based MTJs is that they are all found to exhibit high TMR values, while typically only a few are reported with STO. ${ }^{13,14}$ For example, all measured junctions distributed on the $5 \times 5 \mathrm{~mm}^{2}$ sample with an optimal barrier thickness of $2.6 \mathrm{~nm}$ exhibit TMR values in the 320\%-350\% range at $20 \mathrm{~K}$. In total, $80 \%$ of 40 measured junctions with different barrier thicknesses have shown a TMR of over $100 \%$. Overall, using $\mathrm{Nb}: \mathrm{STO}$ as the insulator results in outstanding, reproducible, and stable junctions.

There are several invaluable features of the Nb:STO insulator that acts as a tunnelling barrier for electrons. ${ }^{7}$ First, the magneto-resistance versus magnetic field curve (Fig. 1(c)) demonstrates the typical TMR behaviour observed in

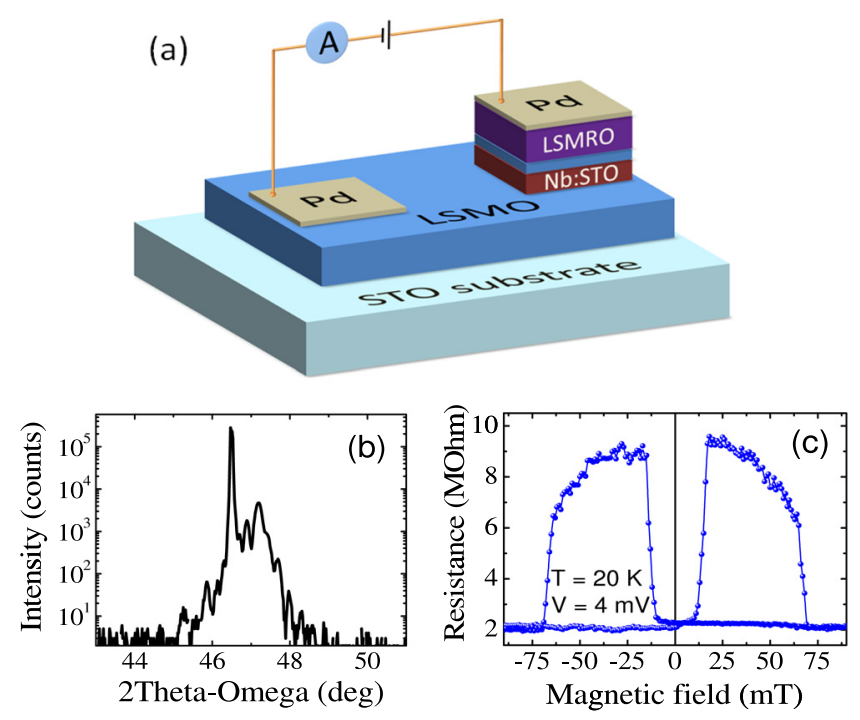

FIG. 1. (a) Schematic of the micrometer-sized MTJ mesa together with Pd contact pads. (b) Theta-2theta X-ray diffraction spectrum of a typical stack. (c) Resistance as a function of the magnetic field, measured at $20 \mathrm{~K}$. junctions with an undoped STO insulator. Moreover, the differential conductance in the parallel state at $300 \mathrm{~K}$ (inset in Fig. 2(a)) shows a parabolic dependence, which can be nicely fit using the Brinkman model for tunneling electrons. ${ }^{15}$

The fit allows the extraction of the barrier height $\phi=1.6 \mathrm{eV}$ (with an asymmetry of $\Delta \phi=0.2 \mathrm{eV}$ ) and thickness $(d=1.7 \mathrm{~nm})$. These parameters are strongly dependent on the quality of the barrier. While being in agreement with those reported on an undoped STO barrier, ${ }^{13}$ the apparent barrier height of Nb:STO was found to be higher, hinting at a reduced concentration of defects in $\mathrm{Nb}$ :STO or/and that the double Schottky barrier is a stronger tunnel barrier than STO. Additional evidence for a reduced concentration of defects is that there is not a large drop of the conductance at zero bias (Fig. 2(a)), also called the "zero bias anomaly" (ZBA), even down to $5 \mathrm{~K}$. The ZBA is often explained by the presence of defects in the barrier or at the interface, which induce states inside the barrier and thus additional conducting channels. ${ }^{16,17}$ The vanishing ZBA is strong evidence for a high quality LSMO/Nb:STO interface and/or of the $\mathrm{Nb}: \mathrm{STO}$ barrier, compared to the undoped-STO case.
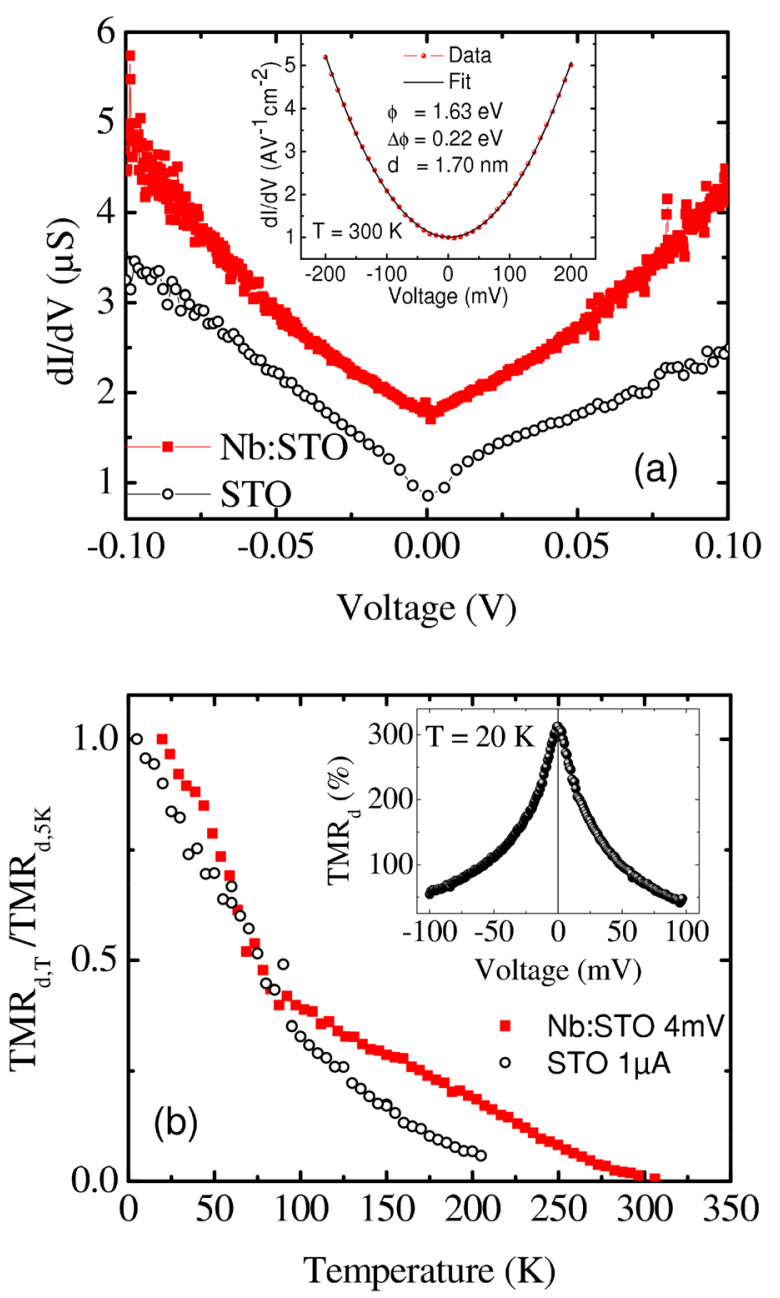

FIG. 2. (a) Differential conductance in the parallel state for STO and $\mathrm{Nb}$ :STO tunnel junctions at $5 \mathrm{~K}$. (Inset) Differential conduction of an $\mathrm{Nb}$ :STO junction at $300 \mathrm{~K}$ with a Brinkman fit. (b) Normalized differential TMR for STO and Nb:STO tunnel junctions as a function of temperature. Note the steep increase in TMR below $100 \mathrm{~K}$ in both systems. (Inset) Bias voltage-dependence of the magneto-resistance in $\mathrm{Nb}$ :STO junctions at $20 \mathrm{~K}$. 


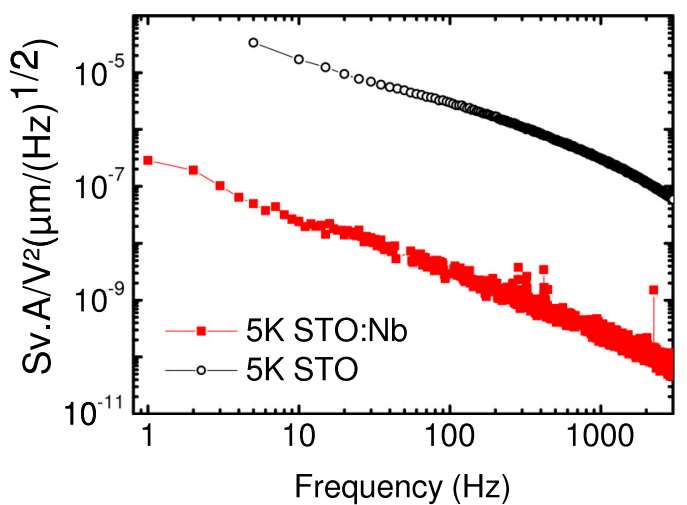

FIG. 3. Voltage noise spectral density at $5 \mathrm{~K}$ in MTJs with an STO (black) and an Nb:STO (red) barrier, respectively. The noise level is reduced by about 3 orders of magnitude in the latter case.

The temperature dependence of the magnetoresistance is similar to that obtained with an undoped STO barrier (Fig. 2(b)). Nevertheless, in Nb:STO junctions, the temperature at which the TMR is extinguished is improved by $100 \mathrm{~K}$, now approaching room temperature. This hints at an improvement of the LSMO magnetic properties at the interface.

The measured TMR ratios are high and similar to the standard STO barrier case, but they do not achieve the two highest published values. ${ }^{14,18}$ The upper bound for the TMR ratio seems thus to depend mostly on the LSMO electrode as it is not noticeably modified by the Nb:STO barrier. As shown in the inset of Fig. 2(b), the TMR is reduced with voltage dependence.

Not only these features but also other core questions merit further investigations, especially the phenomenon of spin-polarized transport through the barrier, which gives rise to the TMR. The landscape of the LSMO density of states (DOS), its dependence on the electric field, and the energy of the tunneling electrons are unknown factors that can all affect the TMR dependence on the barrier thickness, doping level, and applied voltage.

In order to obtain a high performing sensor for applications, the characterization of the noise is essential. The $1 / f$ noise is particularly relevant for device operation at low frequencies. We therefore conducted noise measurements with a low noise amplifier and a battery powered current source. ${ }^{3}$ The noise spectrum of an MTJ exhibits two main contributions: white noise resulting from thermal and shot noise that is independent of frequency and a frequency-dependent term consisting of $1 / f$ noise and random telegraph noise $(\mathrm{RTN})^{19}$ due to resistance fluctuations in the tunnel junction.

Fig. 3 shows that the noise spectral density of LSMO/ $\mathrm{Nb}$ :STO/LSMO junctions in the low frequency range is improved by 3 orders of magnitude compared to the undoped junctions. Previously, the STO barrier was considered as the noise source and explained the noise behavior at low frequency in STO junctions. ${ }^{3}$ Here, the strong noise reduction supports the hypothesis that the barrier has improved properties or less impact on the junction characteristics. The $1 / f$ noise power spectrum can be described by a Hooge like model. ${ }^{20}$ The noise spectral density $S$ can be expressed as

$$
S(f)=\frac{\alpha V^{2}}{A f},
$$

where $A$ is the area of the junction, $V$ is the bias voltage, $f$ is the frequency, and $\alpha$, designated as the Hooge parameter, can be estimated to compare low frequency noise of various devices.

We estimated from the noise spectral density an $\alpha$ of $10^{-6} \mu \mathrm{m}^{2}$ for an $R A$ of $100 \mathrm{M} \Omega \cdot \mu \mathrm{m}^{2}$ reaching the same noise level as in metallic state-of-the-art junctions [Ref. 3 and ref. inside].

In conclusion, we demonstrated significantly improved characteristics of LSMO/STO/LSMO tunnel junctions when undoped STO is replaced with semiconducting Nb:STO.

We have shown that tunnel junctions with an Nb:STO barrier exhibit an enhanced quality with a reduced number of defects, resulting in improved reproducibility of results, large TMR ratios between 100 and $350 \%$ between $20 \mathrm{~K}$ and $100 \mathrm{~K}$, and also a three orders of magnitude improvement of the low frequency noise level. These results open the way to all-oxide sensors for magnetometry applications.

${ }^{1}$ J.-H. Park, E. Vescovo, H.-J. Kim, C. Kwon, R. Ramesh, and T. Venkatesan, Nature 392, 794 (1998).

${ }^{2}$ B. Nadgorny, I. I. Mazin, M. Osofsky, R. J. Soulen, Jr., P. Broussard, R. M. Stroud, D. J. Singh, V. G. Harris, A. Arsenov, and Ya. Mukovskii, Phys. Rev. B 63, 184433 (2001).

${ }^{3}$ R. Guerrero, A. Solignac, C. Fermon, M. Pannetier-Lecoeur, P. Lecoeur, and F. Fernandez-Pacheco, Appl. Phys. Lett. 100, 142402 (2012).

${ }^{4}$ R. P. Borges, W. Guichard, J. G. Lunney, J. M. D. Coey, and F. Ott, J. Appl. Phys. 89(7), 3868-3873 (2001).

${ }^{5}$ T. Tomio, H. Miki, H. Tabata, T. Kawai, and S. Kawai, J. Appl. Phys. 76, 5886 (1994).

${ }^{6}$ M. Minohara, I. Ohkubo, H. Kumigashira, and M. Oshima, Appl. Phys. Lett. 90, 132123 (2007).

${ }^{7}$ G. Kurij, L. E. Calvet, R. Guerrero, T. Maroutian, G. Agnus, A. Solignac, and $\mathrm{Ph}$. Lecoeur, Thin Solid Films 617, 82-85 (2016).

${ }^{8}$ M. Jullière, Phys. Lett. A 54, 225 (1975).

${ }^{9}$ N. Matsuo, N. Doko, T. Takada, H. Saito, and S. Yuasa, Phys. Rev. Appl. 6, 034011 (2016).

${ }^{10}$ R. Waser and D. M. Smyth, "Defect chemistry, conduction, and breakdown mechanism of perovskite-structure titanates," in Ferroelectric Thin Films: Synthesis And Basic Properties, edited by C. P. de Araujo, J. F. Scott, and G. W. Taylor (Gordon and Breach Publishers, Amsterdam, 1996), p. 47.

${ }^{11}$ N.-H. Chan, R. K. Sharma, and D. M. Smyth, J. Electrochem. Soc. 128, 1762 (1981).

${ }^{12}$ A. Solignac, R. Guerrero, G. Agnus, C. Fermon, M. Pannetier-Lecoeur, and Ph. Lecoeur, J. Phys.: Conf. Ser. 303, 012059 (2011).

${ }^{13}$ A. Solignac, G. Kurij, R. Guerrero, G. Agnus, T. Maroutian, C. Fermon, M. Pannetier-Lecoeur, and P. Lecoeur, Proc. SPIE 9551, 95512F (2015).

${ }^{14}$ M. Bowen, M. Bibes, A. Barthelemy, J.-P. Contour, A. Anane, Y. Lemaitre, and A. Fert, Appl. Phys. Lett. 82, 233 (2003).

${ }^{15}$ W. F. Brinkman, R. C. Dynes, and J. M. Rowell, J. Appl. Phys. 41, 1915 (1970).

${ }^{16}$ J. O'Donnell, A. E. Andrus, S. Oh, E. V. Colla, and J. N. Eckstein, Appl. Phys. Lett. 76(14), 1914-1916 (2000).

${ }^{17}$ J. Z. Sun, D. W. Abraham, K. Roche, and S. S. P. Parkin, Appl. Phys. Lett. 73(7), 1008-1010 (1998).

${ }^{18}$ R. Werner, A. Yu. Petrov, L. Alvarez Mino, R. Kleiner, D. Koelle, and B. A. Davidson, Appl. Phys. Lett. 98, 162505 (2011).

${ }^{19}$ C. Fermon and C. M. Pannetier-Lecoeur, "Noise in GMR and TMR Sensors," in Giant Magnetoresistance (GMR) Sensors (Springer, Berlin/ Heidelberg, 2013), pp. 47-70.

${ }^{20}$ F. N. Hooge and A. Hoppenbrouwers, Physica 45, 386-392 (1969). 